\title{
Stage II Hepatocellular Carcinoma AJCC v8
}

National Cancer Institute

\section{Source}

National Cancer Institute. Stage II Hepatocellular Carcinoma A/CC v8. NCI Thesaurus.

Code C134519.

Stage II includes: T2, N0, M0. T2: Solitary tumor larger than $2 \mathrm{~cm}$ with vascular invasion, or multiple tumors, none larger than $5 \mathrm{~cm}$. NO: No regional lymph node metastasis. M0: No distant metastasis. (AJCC 8th ed.) 